\title{
Some Effects of Prenatal Zinc Deficiency on Behavior of the Adult Rat
}

\author{
E. S. HALAS and H. H. SANDSTEAD ${ }^{(31)}$ \\ Department of Psychology, University of North Dakota, Grand Forks, and United States Department of \\ Agriculture, Agriculture Research Service, Human Nutrition Laboratory, Grand Forks, North Dakota, USA
}

\section{Extract}

The effect of zinc deficiency during the latter third of gestation on avoidance conditioning of young adult male rats was compared with the effect of intrauterine starvation and normal pregnancy. Animals which had experienced zinc deficiency avoided shock less well than the offspring of pair-fed control dams. The offspring of pair-fed dams avoided shock less well than animals which were the product of normal pregnancy. Response latencies were inferior in the zinc-deficient group, whereas intertrial responses were less on both the previous zinc-deficient and intrauterine starved animals.

\section{Speculation}

Zinc is essential for development of the brain. Fetuses of women who consume inadequate amounts of zinc during pregnancy may be at risk as far as intrauterine brain development and body growth are concerned. Damage caused to such fetuses may be persistent and be mainfest as mental retardation.

Zinc is essential for nucleic acid synthesis in the rat $(18,19$, $23,25)$ and protein $(8,25)$ and, therefore, presumably in man. Perhaps becuase of its role in these aspects of metabolism, zinc deficiency has adverse effects on the brain of the fetal (9) and suckling (18) rat. The injury to the brain of the suckling rat is apparently poorly reversible, as behavioral tests of 60- to 80 -day-old rats which have been suckled by zinc-deficient dams until weaning have revealed impaired acquisition of the Tolman Honzig maze (12).

The evidence that zinc is essential for muturation of the brain and that zinc deprivation during the critical period of brain growth results in persistent evidence of injury prompted us to assess the effect of intrauterine zinc deficiency during the third trimester of gestation on subsequent avoidance conditioning of 60-day-old rats. Previous evidence that intrauterine malnutrition can cause persistent behavioral abnormalities in adult rats has been limited (21).

\section{MATERIALS AND METHODS}

Timed pregnant Sprague-Dawley rats were divided into three groups and individually housed in plastic cages with perforated bottoms in a light- and temperature-controlled environment. The dams were purchased timed pregnant from BioLabs (26) and the day of mating was counted as day 0 . From the 15 th through the 20th day of gestation they were fed a sprayed egg white, biotin-enriched diet containing $<1,0 \mathrm{ppm}$ zinc (13). During this interval two groups served as controls. One control group (five dams) was pair-fed with the zinc-deficient group, while the second control group (five dams) was fed the diet ad libitum. Both control groups were given drinking water containing $50 \mathrm{ppm}$ zinc via an automatic watering device. The zinc-deficient group (five dams) were given trace mineral-free water via a central watering device. Before and after the "zinc-deficient interval," all dams were fed a standard laboratory rat chow ad libitum. Food consumption and weight gain of the dams were recorded during pregnancy and lactation. After delivery the number of pups per litter was made approximately equivalent. All litters thus contained 8-10 pups and paired dams nursed the same number of pups. The pups were weaned on the 21 st postnatal day and fed chow. The weight gains of the pups were recorded from the 1 st through the 40th day of life. Their food consumptions were recorded from the 21 st through the 40 th day of life.

Behavioral testing was started when the rats were 60 days old. Ten male rats were selected randomly from the litters of each group and all 30 rats were given the following behavioral training. The rats were given 25 trials $/ 24 \mathrm{hr}$ for 3 days of habituation to a conditioned stimulus (CS) in a two-way shuttle box (27). The box was placed in an air conditioned, sound insulated, and lightproof environment with a small one-way mirror. The CS consisted of a $28-\mathrm{V}$ light presented for $20 \mathrm{sec}$. The intertrial period lasted $50 \mathrm{sec}$. The number and latency of jumps across the barrier were automatically recorded during the CS period and the intertrial period.

After habituation, the rats were given 25 trials $/ 24 \mathrm{hr}$ of avoidance conditioning for 5 days. If the rat jumped the barrier during the first $5 \mathrm{sec}$ of the CS, no shock was given. If the rat did not jump the barrier within $5 \mathrm{sec}$, a 1.0-ma shock was given during the remainder of the CS period $(15 \mathrm{sec})$ or until the rat jumped the barrier. Jumping the barrier terminated the shock. The number and latency of all jumps during the avoidance trial and the intertrial period were recorded by a remote automatic device. At the completion of the avoidance training, all rats were given 25 trials $/ 24 \mathrm{hr}$ of extinction for 2 days. No shock was given at any time during the 20 -sec period.

\section{RESULTS}

Figure 1 shows the food consumption of the dams during the time they were fed the zinc-deficient diet during the latter one-third of pregnancy and their consumption of chow during the first half of the suckling period. Figure 2 shows their weights during the same intervals. The non-zinc supplemented dams showed loss of appetite. Because one of the earliest effects of zinc deficiency is anorexia, the decrease in food consumption of the non-zinc-supplemented dams provided 


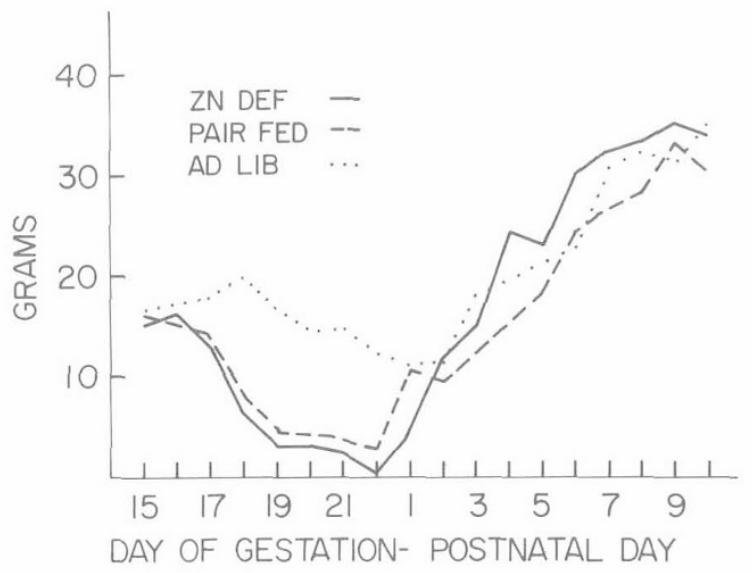

Fig. 1. Mean food consumption of dams. The zinc-deficient $(Z N$ $D E F$ ) dams demonstrated a decreased food consumption which was clearly evident by the 17th day of gestation. By the second postnatal day food consumption of all groups was similar.

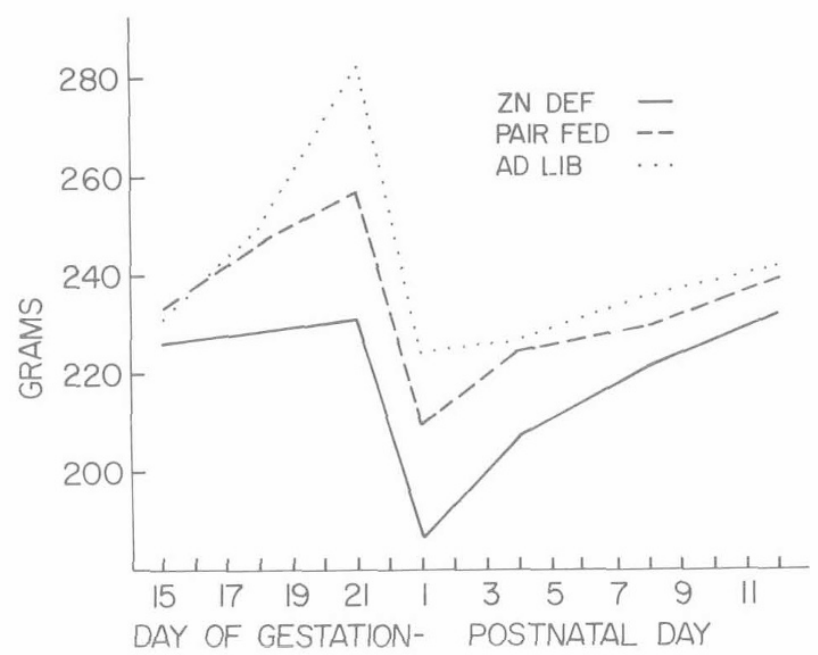

Fig. 2. Mean body weights of dams, Zinc-deficient (ZN DEF) dams showed almost no weight gain after initiation of the zinc-deficient diet. The better food utilization of the pair-fed controls was reflected by their increase in weight, although the amount of food they consumed was similar to that eaten by zinc-deficient dams. All of the animals gained weight postnatally, which indicated that the dietary intake of essential nutrients was adequate.

evidence that they were indeed zinc deficient (3). The poor weight gain of the non-zinc-supplemented group is additional evidence for deficiency. The pair-fed dams showed greater weight gains even though their food intake had been similar to that of the non-zinc-supplemented group. The poorer feed efficiency of the latter group is also evidence that they were zinc deficient.

Because of warm-up effects, the first five trials of each day's block of 25 trials were eliminated from the figures and the statistical analysis. The remaining 20 trials for each day were divided into two groups of 10 trials for both the figures and statistical analysis.

The effects of intrauterine zinc deficiency on avoidance conditioning of the offspring are shown in Figure 3. During habituation, all groups responded equally to the CS. However, during avoidance conditioning the previously zinc-deficient animals learned to avoid the shock less well than either control group. A two-way analysis of variance was computed and found to be significant $(\mathrm{F}=14.16$, df 2,$90 ; P<0.001)$. Therefore $t$ tests were calculated between the zinc-deficient

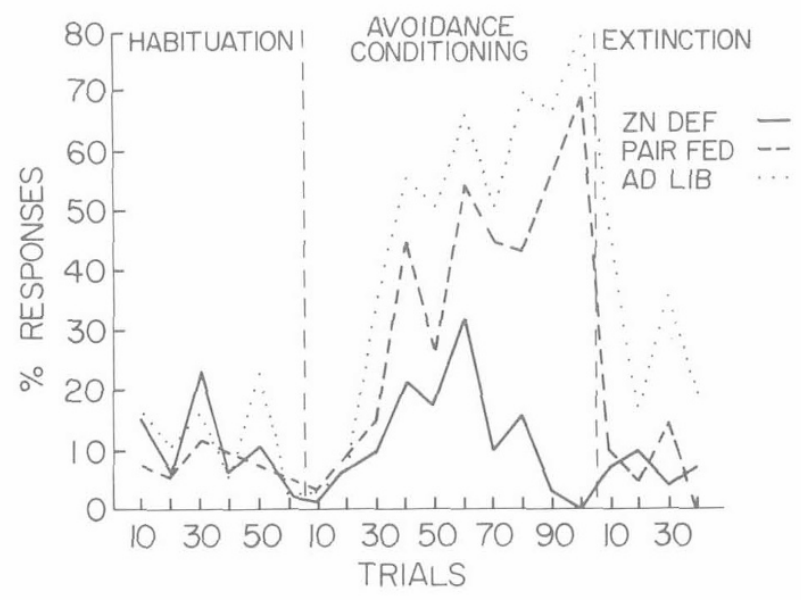

Fig.3. Avoidance conditioning, percentage response. All three groups were equivalent during the habituation trials. However, during the avoidance conditioning trials, the in utero zinc-deficient $(Z N D E F)$ rats reached their peak level of performance on the 60th trial and then declined, whereas the pair-fed and ad libitum rats continued to improve (although the ad libitum group was superior). During the extinction period the avoidance of the pair-fed group dropped very rapidly to the level of the zinc-deficient group. Each point on the figure represents the mean of 10 animals for a block of 10 trials.

and ad libitum groups $(t=3.082, \mathrm{df}=20, P<0.001)$; between the zinc-deficient and pair-fed groups $(t=2.85$, df $20 ; P<$ $0.01)$, and between the ad libitum and pair-fed groups $(t=$ 2.45 , df $20 ; P<0.05)$. In addition, the members of the previously zinc-deficient group demonstrated a peak and then decline in their avoidance suggesting that they may have been unusually sensitive to shock. This decrement in performance is generally interpreted as evidence of an inability to tolerate stress. In contrast to the animals which had experienced intrauterine zinc deprivation, those animals whose dams had been pair-fed (starved) during the third trimester learned to avoid the shock better although somewhat less well than did the controls whose dams have been fed ad libitum throughout pregnancy.

The animals whose dams had been deprived of zinc or whose dams had been pair-fed (starved) both extinguished the avoidance response more rapidly than did the animals whose dams were adequately fed throughout pregnancy. The overall $F$ ratio was 10.56 , df 2,$90 ; P<0.001$. The $t$ test between the zinc-deficient and ad libitum groups was 4.26, df $20 ; P<$ 0.001 . Between the pair-fed and ad libitum groups the $t$ test was 5.95 , df $20 ; P<0.001$. There was no significant difference between the pair-fed and zinc-deficient groups $(t=1.69$, df 20 ; $P>0.10)$. These observations suggest that the level of avoidance conditioning reached by the ad libitum group was so high that members of the group were unable to discriminate the change in the experimental conditions that occurred after avoidance conditioning was stopped and extinction was begun. The data may be interpreted to indicate that the apparently inferior learning shown during avoidance conditioning by the animals whose dams had been pair-fed (starved) during the latter one-third of pregnancy represents a real difference between them and the animals whose dams had been adequately fed.

The response latencies again illustrate the persistent effects of the zinc deficiency. All three groups showed no differences in latencies during habituation (Figure 4). However, during avoidance conditioning, the zinc-deficient group was significantly slower to respond than the two control groups. The F ratio was 26.99 , df 2,$90 ; P<0.001$. The $t$ test between the zinc-deficient and ad libitum group was 4.09 , df $20 ; P<$ 0.001 , whereas between the zinc-deficient and pair-fed groups it was 3.13 , df $20 ; P<0.01$. The latencies of the animals 
whose dams had been pair-fed were slightly longer than those whose dams had been adequately fed; however, the difference was not significant $(t=0.93)$. During the extinction period (no shock) animals whose dams had been fed ad libitum showed significantly shorter latencies.than either of the other two groups of animals $(\mathrm{F}=5.56$, df 2,$90 ; P<0.01)$. The $t$ test between the ad libitum and zinc-deficient group was 3.02 , df $20 ; P<0.01$, and the $t$ test between the ad libitum and pair-fed was 2.20 , df $20 ; P<0.05$. There was no significant difference between the pair-fed and zinc-deficient groups.

The intertrial responses (Fig. 5) indicate that the animals whose dams had been zinc deficient during the latter one-third of pregnancy and the aminals whose dams had been pair-fed (starved) during that interval of pregnancy were less active than those whose dams had been adequately fed throughout pregnancy. The $\mathrm{F}$ ratio was 23.18 , df 2 , 90; $P<0.001$.

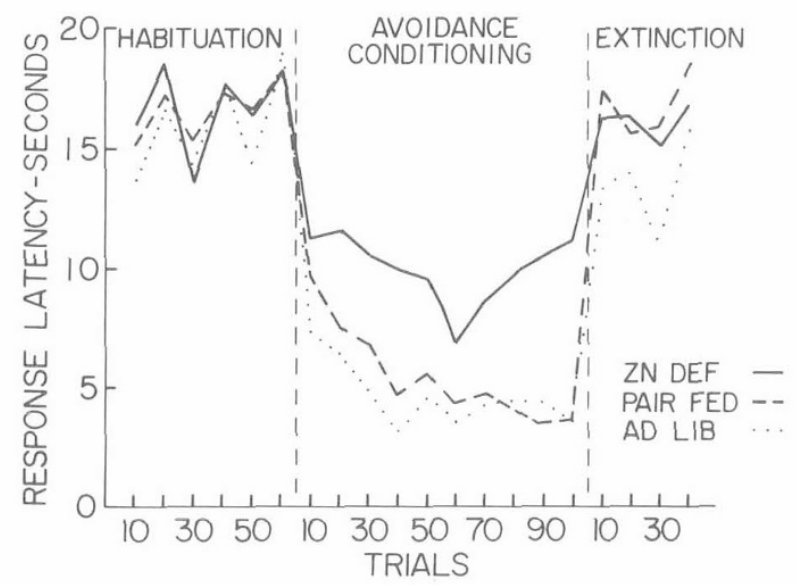

Fig. 4. Avoidance conditioning response latency. The response latency also illustrates the inferior performance of the zinc-deficient (ZN DEF) group during avoidance conditioning as compared with the two control groups. During extinction, the ad libitum group responded more quickly to the Conditioned Stimulus. Each point on the figure represents the mean of 10 rats for a block of 10 trials.

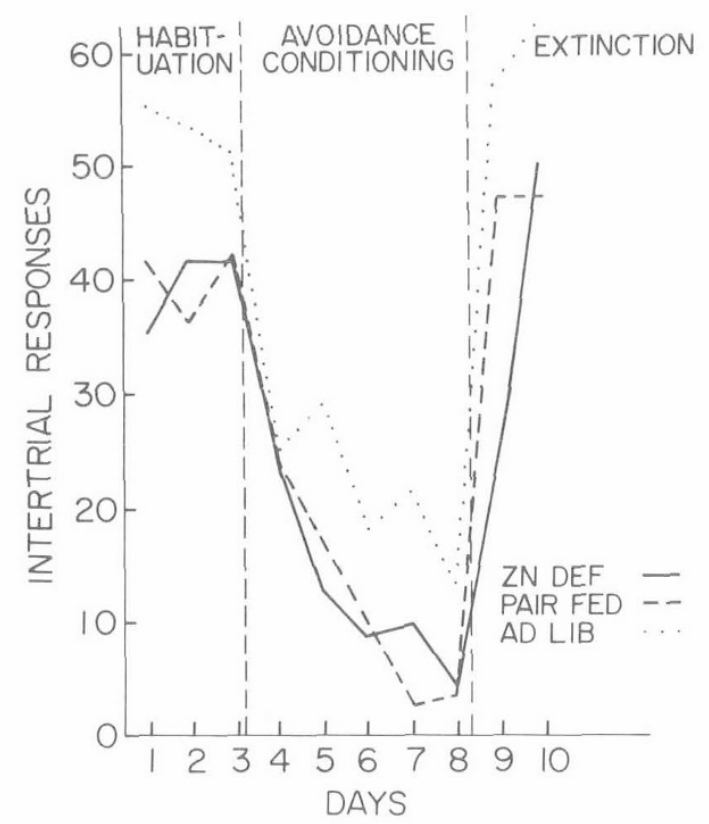

Fig. 5. Avoidance conditioning, intertrial responses. Both the zinc-deficient $(Z N D E F)$ and pair-fed groups were less active than the ad libitum group during habituation, avoidance conditioning, and extinction. Each point on the figure represents the mean of 10 animals for a block of 25 trials.
Therefore, $t$ tests were computed between ad libitum and zinc-deficient groups $(t=4.02$, df $20 ; P<0.001)$ and also between ad libitum and pair-fed groups $(t=4.85$, df $20 ; P<$ $0.001)$. No significant difference was found between the two groups of intrauterine deprived animals $(t=1.09$, df $20 ; P>$ $0.05)$.

\section{DISCUSSION}

The results of the experiment reported here suggest that both intrauterine zinc deficiency and intrauterine starvation during the latter one-third pregnancy have long term adverse effects on the behavior of the rat. The effects of zinc deficiency appear to be more severe than those of starvation alone.

The rats which had been deprived of zinc during the latter one-third pregnancy performed poorly and reached a peak of only $35 \%$ avoidance responses midway through the experiment. This was then followed by a marked decrement in response. The decrement probably was due to an inability to tolerate the stress induced by the level of shock used. One milliampere is near the threshold of shock tolerated by normal rats. When normal rats are subjected to levels of shock beyond their threshold, their performance becomes subnormal and is followed by a marked decrement $(1,2,11,14,16,24)$. The response latency of the zinc-deficient group was significantly longer than of either of the control groups. This finding supports the interpretation that the animals which had been exposed to intrauterine zinc deficiency were inferior in their ability to learn to avoid the shock. The level of activity of the intrauterine zinc-deprived group as measured by the intertrial responses was less than that for normal control groups but was equal to that of the rats born to the pair-fed (starved) dams, which suggests zinc deficiency in utero did not have adverse effects on activity which were different than the effects of intrauterine starvation alone.

The rats whose dams were pair-fed (starved) during the latter one-third of pregnancy did learn to avoid the shock $70 \%$ of the time. However, their performance during extinction suggests that they readily discriminated the change from shock during conditioning to no shock during extinction and therefore stopped making avoidance responses. In contrast, the normal rats achieved an $80 \%$ level of avoidance and during the no shock situation (extinction) did not readily distinguish from the shock situation during conditioning. Thus the normal rats extinguished more slowly than the rats which had experienced intrauterine starvation $(P<0.001)$. This finding supports the observation that the level of learning reached by the normal rats was greater than that of the offspring of the pair-fed dams $(P<0.05)$. The response latency of the intrauterine starved rats was somewhat, but not significantly longer than that of the normal rats.

For technical reasons the litters were not cross-fostered to normal dams in this experiment. Therefore, it is not possible to rule out some impairment in lactational ability in the dams which had been exposed to zinc deficiency or pair feeding. Inspection of Figure 1 shows that once rehabilitation was started, the food consumption of the zinc-deficient dams rapidly caught up and slightly exceeded the other groups. In addition, the weight gain of the zinc-deficient dams (Fig. 2) also indicated that rehabilitation of the dams was rapid. Pups nursed by the previously zinc-deficient and pair-fed dams also grew well. Therefore we believe the major injury occurred during intrauterine life and postnatal effects, while possible, were not a major factor in brain injury. Other research in our laboratory has shown that intrauterine zinc deficiency from the 15 th through the 21 st day of gestation impairs the growth of the fetus, while pair feeding during this interval has a lesser effect (15).

In summary, the data are consistent with the hypothesis 
that zinc deficiency during the latter one-third of pregnancy will significantly retard the subsequent ability of male rats to learn an avoidance response as young adults. The data also indicate that zinc deficiency during the latter one-third of gestation has adverse effects on the ability of the male rat to tolerate stress as a young adult. Other effects include an apparent decrease in physical activity. Rats exposed to intrauterine starvation, but not to zinc deficiency also show a reduction in their ability to learn an avoidance response as adults. However, they do perform significantly better than those which have been exposed to zinc deficiency. Their activity appears to be decreased to a level similar to that of the previously zinc-deficient animals. On the other hand, intrauterine starvation during the third trimester does not appear to affect the ability of rats to tolerate a 1.0 -ma stress. The adverse effects of zinc deficiency in utero are clearly more severe than those of intra uterine starvation alone.

The findings in the study reported here may have relevance to man for two reasons. The least well understood, but perhaps more important reason is the evidence that some women may not ingest adequate amounts of zinc during pregnancy. This interpretation is based on calculations of probable requirements and diet analysis (17), on the reported finding of decreased levels of zinc in plasma during the latter part of pregnancy $(6,7)$, and on the finding that concentrations of zinc in hair during pregnancy and lactation change in a way which is consistent with a decrease in the body stores of zinc (10). The second reason is the observation that infants with severe protein-energy malnutrition may have an associated zinc deficiency $(20,22)$.

Although it is unknown at present whether zinc deficiency in the human fetus or young infant has adverse effects on the brain, the findings in this and other studies $(5,12,18)$ suggest that the question may be worthy of investigation. Until data is forthcoming, these findings suggest that it may be prudent in the meantime to assure an adequate intake of zinc during human pregnancy.

Although this study was not concerned with the mechanism of the observed effect, it is possible to suggest some of the biochemical consequences of zinc deprivation which may have been responsible for the findings. Zinc is essential for nucleic acid, protein, cerebroside, and lipid synthesis in the brain (4, $5,18)$. Postnatal zinc deficiency severely retards biochemical development of the brain $(4,5)$. It seems highly probable that zinc deficiency has similar effects on the brain of the fetus during the latter one-third of pregnancy. In contrast to zinc deficiency, intrauterine starvation had less severe effects. Presumably this was due to the fact that all necessary substrates for brain growth were present in correct proportions one to another, although in decreased amounts. Thus, the growth and biochemical maturation which did occur were not distorted by the absence of a single critical nutrient.

\section{REFERENCES AND NOTES}

1. Bauer, R. H.: The effects of CS and US intensity on shuttlebox avoidance. Psychon. Sci., 27: 266 (1972).

2. Bintz, J.: Effect of shock intensity on the retention of an avoidance response. Psychon. Sci., 22: 17 (1971). 22: 17 (1971).

3. Chesters, J. K., and Quarterman, J.: Effects of zinc deficiency on food intake and feeding patterns of rats. Brit. J. Nutr., 24: 1061 (1970).

4. Fosmire, G. J., Al-Ubaidi, Y. Y., Halas, E. S., and Sandstead, H. H.: The effect of zinc deprivation on the brain. In: M. Friedman: Protein-Metal Interactions, p. 329 (PIenum Publishing Corp., New York, 1974).
5. Fosmire, G. J., and Sandstead, H. H.: Some effects of postnatal zinc deficiency on developing rat brain. Pediat. Res. 9: 00 (1975).

6. Halsted, J. A., and Smith, J. C., Jr.: Plasma-zinc in health and disease. Lancet, $i$ : 322 (1970).

7. Henkin, R. I.: Newer aspects of copper and zinc metabolism. In: W. Mertz and W. Cornatzer: Newer Trace Elements, p. 255 (Marcel Dekker, New York, 1970).

8. Hsu, J. M., Anthony, W. L., and Buchanan, P. J.: Zinc deficiency and incorporation of ${ }^{24} \mathrm{C}$-labeled methionine into tissue proteins in rats. J. Nutr., 99: 425 (1969).

9. Hurley, L. S.: Zinc deficiency in the developing rat. Amer. J. Clin. Nutr., 22: 1332 (1969).

10. Klevay, L. M.: Hair as a biopsy material. I. Assessment of zinc nutriture. Amer. J. Clin. Nutr., 23: 284 (1970).

11. Levine, S.: UCS intensity and avoidance learning. J. Exp. Psychol.., 71: 163 (1966).

12. Lokken, P. M., Halas, E. S., and Sandstead, H. H.: Influence of zinc deficiency on behavior. Proc. Soc. Exp. Biol. Med., 144: 680 (1973).

13. Luecke, R. W., Olman, N. E., and Baltzer, B. V.: Zinc deficiency in the rat: Effect on serum and intestinal alkaline phosphatase activities. J. Nutr., 94: 344 (1968).

14. McAllister, W. R., McAllister, D. E., and Douglas, W. K.: The inverse relationship between shock intensity and shuttle-box avoidance learning in rats: A reinforcement explanation. J. Comp. Physiol. Psychol., 74: 426 (1971).

15. McKenzie, J. M., Fosmire, G. J., and Sandstead, H. H.: Zinc deficiency in the prenatal rat. Proceedings of the North Dakota Academy of Sciences, in press.

16. Moyer, K. E., and Korn, J. H.: Effect of UCS intensity on the acquisition and extinction of an avoidance response. J. Exp. Psychol., 67: 352 (1964).

17. Sandstead, H. H.: Zinc nutrition in the United States. Amer. J. Clin. Nutr, 26: 1251 (1973).

18. Sandstead, H. H., Gillespie, D. D., and Brady, R. N.: Zinc deficiency: Effect on brain of the suckling rat. Pediat. Res., 6: 119 (1972).

19. Sandstead, H. H., and Rinaldi, R. A.: Impairment of deoxyrioonucleic acid synthesis by dietary zinc deficiency in the rat. J. Cell Physiol., 73: 81 (1969).

20. Sandstead, H. H., Shukry, A. S., Prasad, A. S., Gabr, M. K., El Hefny, A., Mokhtar, N., and Darby, W. J.: Kwashiorkor in Egypt. I. Clinical and biochemical studies with special reference to plasma zinc and serum lactic dehydrogenase. Amer. J. Clin. Nutr., 17: 15 (1965).

21. Smart, J. L., Dobbing, J., Adlard, B. P. F., Lynch, A., Sands, J.: Vulnerability of developing brain: Relative effects of growth restriction during the fetal and suckling periods on behavior and brain composition of adult rats. J. Nutr., 103: 1327 (1973).

22. Smit, Z. M., and Pretorius, P. J.: Studies in metabolism of zinc. Part 2: Serum zinc leveis and urinary zinc excretions in South African Bantu kwashiorkor patients, J. Trop. Pediat., 9: 105 (1964).

23. Terhune, M. W., and Sandstead, H. H.: Decreased RNA polymerase activity in mammalian zinc deficiency. Science, 177: 86 (1972).

24. Theios, J., Lynch, A. D., and Lowe, W. F., Jr.: Differential effects of shock intensity on one-way and shuttle avoidance conditioning. J. Exp. Psychol., 72: 294 (1966).

25. Williams, R. B., Chesters, J. K.: The effects of early zinc deficiency on DNA and protein synthesis in the rat. Brit. J. Nutr., 24: 1053 (1970).

26. Mention of a trademark or proprietary product does not constitute a guarantee or warranty of the product by the United States Department of Agriculture, and does not imply its approval to the exclusion of other products that may also be suitable.

27. Lehigh Valley no. 146-04, Lehigh Valley Co., Lehigh, Penn.

28. Dr. E. S. Halas is Professor, Department of Psychology, University of North Dakota.

29. Dr. H. H. Sandstead is Director, United States Department of Agriculture, Agricultural Research Service, North Central Region, Human Nutrition Laboratory.

30. This research was supported in part by United States Department of Agriculture Cooperative Agreement 12-14-100-11, 178 (61), Amendment 1 .

31. Requests for reprints should be addressed to: H. H. Sandstead, M.D., United States Department of Agriculture, Agricultural Research Service, North Central Region, Human Nutrition Laboratory, 2420 2nd Ave. North, Grand Forks, N. C. 58201 (USA).

32. Accepted for publication September 6, 1974. 